\title{
Comparative Study on Catalytic Dehydration of Glycerol with Alcohols
}

\author{
Guangxin Jia, ${ }^{\circledR *, a}$ Wenlin Ma, ${ }^{a}$ Beibei He, ${ }^{a}$ Yifan Sun ${ }^{a}$ and Laishuan Liu ${ }^{a}$ \\ ${ }^{a}$ School of Chemical Engineering and Technology, North University of China, 030051 Taiyuan, China
}

A comparative study of glycerol dehydration with alcohols (methanol, ethanol, tert-butyl alcohol) over ion exchange resin Amberlyst-35 was performed. The effect of catalyst amounts (1-15 wt.\% to glycerol), reaction temperature $\left(50-100{ }^{\circ} \mathrm{C}\right)$, reaction time $(5-10 \mathrm{~h})$, and the molar ratio of alcohols to glycerol (3:1-8:1) were carried out in $50 \mathrm{~mL}$ stainless steel autoclave. During the process of glycerol dehydration with alcohols, when the catalyst amount (wt.\% to glycerol) is over $10 \%$, it was observed that neither glycerol conversion nor product selectivities are changed significantly. The optimum molar ratios of glycerol to methanol, ethanol and tert-butyl alcohol were $1: 6,1: 4$, and 1:6, respectively. The appropriate reaction temperature and reaction time were $80^{\circ} \mathrm{C}$ and $8 \mathrm{~h}$ at which the selectivities of di-alkyl glycerol ethers and tri-alkyl glycerol ether arrive to the highest. However, the main product was mono-alkyl glycerol ether (MAGE) in glycerol dehydration with tert-butyl alcohol due to steric hindrance influence. If MAGE is considered as the target product, the glycerol dehydration with tert-butyl alcohol could be a good choice. Additionally, the glycerol dehydration with ethanol could be a better route converting biomass material into vehicle fuel in view of its desired yields of higher ethers.

Keywords: alcohols, glycerol, catalytic, dehydration, liquid phase

\section{Introduction}

The excessive consumption of fossil fuels has forced scientists to search for an alternative energy source. On account of the cleanability and renewability, biodiesel is considered as an attractive substituting energy source and this leads to a surplus of glycerol. Therefore, it is very necessary to convert the surplus glycerol into high added value chemicals, which has attracted more and more attention from the world.

In the synthesis routes of glycerol into high value chemicals (such as glycol, acrolein, 1,3-propanediol, 1,2-propanediol), ${ }^{1-3}$ the synthesis of alkyl glycerol ethers has been a hot topic. The alkyl glycerol ethers include mono-alkyl glycerol ethers (MAGE), di-alkyl glycerol ethers (DAGE) and tri-alkyl glycerol ethers (TAGE).

In view of the special biological properties, the MAGE can be used in immunostimulation, ${ }^{4}$ antimicrobial,, 5 anti-tumor activity ${ }^{7-9}$ or equine anesthesia. ${ }^{10}$ Moreover, it is used in cosmetics, ${ }^{11,12}$ such as hair dyes, shampoos, skin creams and deodorants. ${ }^{13-19}$ The DAGE and TAGE are mainly used as diesel additive, especially the TAGE. They can remarkably reduce the emissions of particles, hydrocarbons, carbon monoxide and irregular aldehydes.

*e-mail: jiaguangxin@nuc.edu.cn
TAGE can also be used as low temperature flow improver to reduce diesel viscosity. ${ }^{20-22}$

The synthesis routes of alkyl glycerol ethers mainly are the etherification of glycerol with olefin (isobutene) and the dehydration of glycerol with alcohols (methanol, ethanol, tert-butyl alcohol). However, the olefin polymerization present in the former can cause poor aim-product selectivities. ${ }^{23}$ In a study of Karinen and Krause,${ }^{24}$ they tried to prevent the isobutene polymerization by adding a certain mass fraction of tert-butyl alcohol in the raw materials. Their result indicated that the methyl tert-butyl ether (MTBE) selectivity increased to $97 \%$ over wet ion exchange resin Amberlyst-35.

Recently, the synthesis of poly alkyl glycerol ethers (DAGE and TAGE) has been paid more attention. Its generation process was shown in Figure 1.

Pariente et al. ${ }^{25}$ studied the etherification of glycerol and ethanol over high silicon zeolite molecular sieves and ion exchange resin. The glycerol conversion was 54\% over BEA 12.5 zeolite catalyst. According to Yuan et al. ${ }^{26}$ they investigated the performance of phosphotungstic acid (HPW) catalyst to catalyze the glycerol etherification with ethanol. The results indicated that the catalytic effect of HPW and $\mathrm{HPW} / \mathrm{SiO}_{2}$ was the best, and the glycerol conversion could reach $97.1 \%$. Sepúlveda et al..$^{27}$ compared the glycerol etherification with tert-butyl alcohol by two 


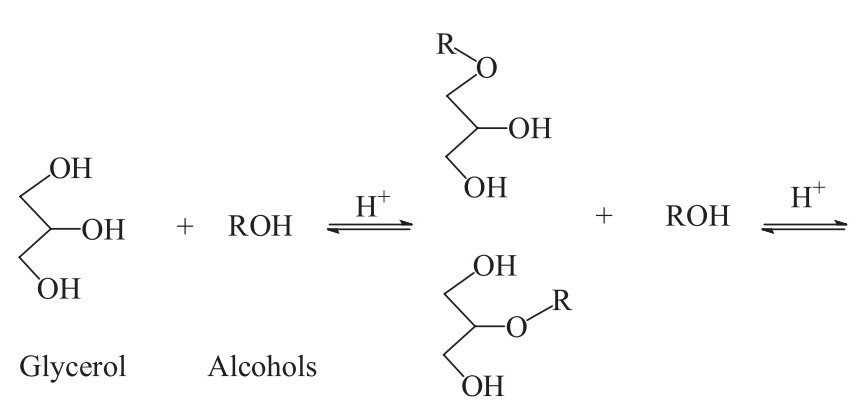

MAGE

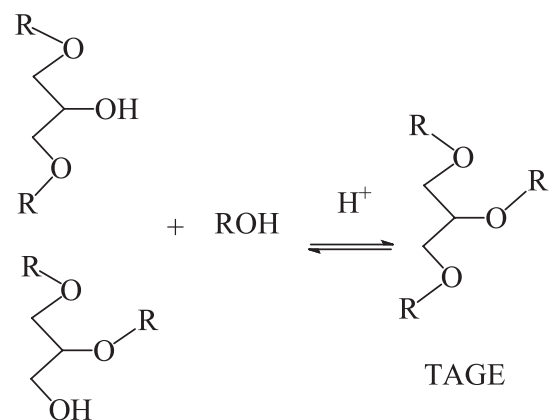

DAGE

Figure 1. The dehydration process of glycerol with alcohols.

catalysts, HPW and ion exchange resin Amberlyst-35 (A-35).

Although there were still many reports ${ }^{28-33}$ about the dehydration of glycerol with alcohols, few researches about the comparative analysis of glycerol dehydration with alcohols were studied.

Generally, the main catalysts for dehydration of glycerol with alcohols are liquid and solid acids. However, liquid acid catalyst has some problems such as product separation difficulty, equipment corrosion, and environment pollution. Therefore, it is a good choice to employ solid acid as the dehydration catalyst of glycerol with alcohols.

In this paper, glycerol and alcohols (methanol, ethanol and tert-butyl alcohol) were selected as the raw materials. The ion exchange resin Amberlyst-35 was used as catalyst, and the effects of the molar ratio of raw materials, different catalyst amounts, reaction temperature and time on the synthesis of methyl glycerol ethers were studied in a stainless steel autoclave. Moreover, the results were also compared and analyzed. It is expected that this research would provide a reference for the further utilization of glycerol.

\section{Experimental}

\section{Chemical reagents and materials}

All chemical reagents used in the paper are analytical reagent grade, which were: glycerol (Tianjin Fuyu Fine chemical Co., Ltd.), methanol (Tianjin Chemical Reagents Plant), ethanol (Shanghai Aibi Chemical Reagents Co., Ltd.), and tert-butyl alcohol (Tianjin Kaitong Chemical Reagents Co., Ltd.).

Ion exchange resin Amberlyst-35 (A-35wet) was purchased from Cmallegro Industrial Co., Ltd., and it was calcined at $100{ }^{\circ} \mathrm{C}$ for $2 \mathrm{~h}$ to obtain A-35dry before used. The physical properties of the catalyst were shown in Table 1.
Table 1. Physical properties of the catalyst

\begin{tabular}{lc}
\hline Property $^{\mathrm{a}}$ & $\begin{array}{c}\text { Ion exchange resin } \\
\text { Amberlyst-35 (dry) }\end{array}$ \\
\hline Exchange capacity / (meq $\left.\mathrm{H}^{+} \mathrm{g}^{-1}\right)$ & 5.2 \\
Surface area / $\left(\mathrm{m}^{2} \mathrm{~g}^{-1}\right)$ & 50 \\
Average pore diameter / $\mathrm{nm}$ & 31 \\
Particle diameter / mm & $0.7-0.95$ \\
Maximum operating temperature $/{ }^{\circ} \mathrm{C}$ & 150 \\
\hline
\end{tabular}

ata obtained from CMALLEGRO industrial Co., Ltd. ${ }^{34}$

\section{Synthesis of alkyl glycerol ethers}

The alcohols, glycerol, solid catalyst as the certain ratio were put into a $50 \mathrm{~mL}$ stainless steel autoclave, then the autoclave was set in a constant temperature oil bath (Chengdong Xinrui Instrument Factory) equipped with a magnetic stirring (70 rpm), and the reaction was studied at a certain temperature from 50 to $100{ }^{\circ} \mathrm{C}$ for $5 \mathrm{~h}$. After reaction, the autoclave was cooled to room temperature and $0.2 \mu \mathrm{L}$ of sample was extracted by microliter syringe (Shanghai Gaoge Industrial and Trading Co., Ltd.) and analyzed by the flame ionization detector (FID) in gas chromatograph (Shimadzu, GC5890) equipped with a capillary column (WONACAP 5, $30 \mathrm{~m} \times 0.32 \mathrm{~mm} \times 0.25 \mu \mathrm{m})$.

The analysis conditions were as follows: $50{ }^{\circ} \mathrm{C}$ for 2 min, heating at $30{ }^{\circ} \mathrm{C} \min ^{-1}$ until $240{ }^{\circ} \mathrm{C}$, with a hold time of $2 \mathrm{~min}$, then heating at $35^{\circ} \mathrm{C} \mathrm{min}^{-1}$ to $300{ }^{\circ} \mathrm{C}$ and holding for $5 \mathrm{~min}$.

The conversion of glycerol and product selectivity were calculated as the follows:

Glycerol conversion $(\%)=\frac{\text { mols glycerol reacted }}{\text { mols glycerol fed }} \times 100$

Product selectivity $(\%)=\frac{\text { mols of product obtained }}{\text { mols of reacted glycerol }} \times 100$ 


\section{Results and Discussion}

Comparative study of physical properties of reactants and products

Methanol, ethanol and tert-butyl alcohol were employed with glycerol to synthesize, respectively, methyl glycerol ether (mono-methyl glycerol ether, di-methyl glycerol ether, tri-methyl glycerol ether), ethyl glycerol ether (mono-ethyl glycerol ether, di-ethyl glycerol ether, tri-ethyl glycerol ether) and tert-butyl glycerol ether (mono-tertbutyl glycerol ether, di-tert-butyl glycerol ether, tri-tertbutyl glycerol ether). The physical properties of reactants and products were shown in Table 2.

Table 2. Physical properties of reactants and products

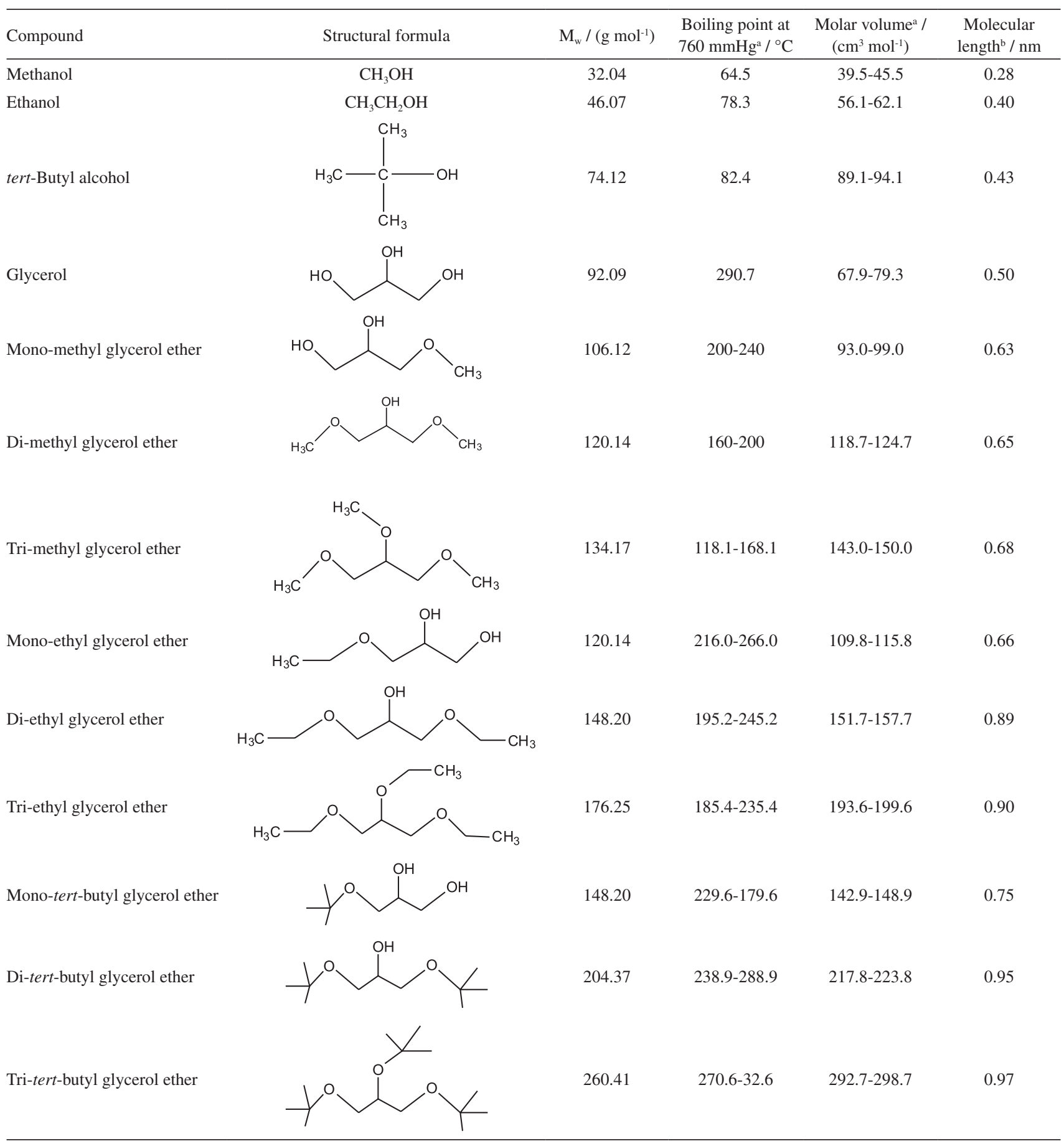

aredicted by the ACD/lab; ${ }^{35}$ bestimated by the ChemBioOffice 2014 software. ${ }^{36} \mathrm{M}_{\mathrm{w}}$ : molecular weight. 
Molar volume and boiling point were predicted by the ACD/labs. ${ }^{35}$ Molecular length was estimated by the ChemBioOffice 2014 software. ${ }^{36}$ As seen in Table 2, among all of products, the tri-tert-butyl glycerol ether owns the greatest molar volume $\left(292.7-298.7 \mathrm{~cm}^{3} \mathrm{~mol}^{-1}\right)$ and molecular length $(0.97 \mathrm{~nm})$. The same result has also been proved by the previous studies, ${ }^{32,37}$ and they have obtained that steric hindrance phenomena could have an impact on the formation of tri-substituted ether and mono-tert-butyl glycerol ether (MTGE) was the main product.

\section{Comparative study of catalyst amount (wt.\% to glycerol)}

The dehydration of glycerol with alcohols (methanol, ethanol and tert-butyl alcohol) over Amberlyst-35 was studied at reaction temperature of $80^{\circ} \mathrm{C}$, reaction time of $8 \mathrm{~h}, 6: 1$ molar ratio of alcohols to glycerol, and stirring speed of $70 \mathrm{rpm}$. The catalyst amount ranges from 1 to 15 wt.\%, and the results were shown in Figure 2.

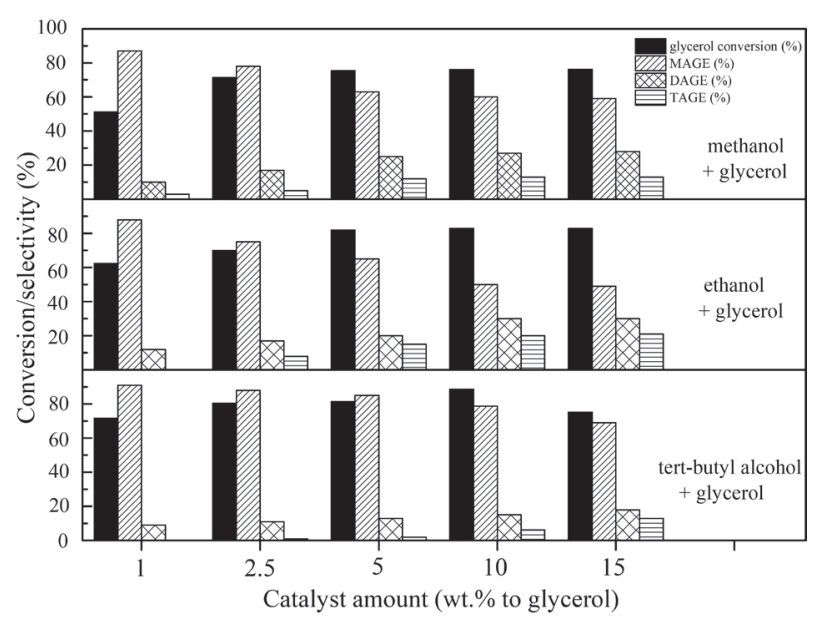

Figure 2. The effect of catalyst amount (wt.\% to glycerol) on catalysis performance.

Under these reaction conditions, the glycerol mainly reacts with alcohols. There are almost no polyglycerols and other by-products detected in this work. The main products are MAGE, DAGE and TAGE.

From Figure 3, when the methanol and glycerol are selected as the raw materials, the glycerol conversion increases with the catalyst amount increase from 1 to $5 \%$, while an increase of catalyst amount from 5 to $15 \%$ does not bring a distinct change in the glycerol conversion, as well as in the product selectivity. In case of the catalyst amount less than 10\%, it can be seen that the selectivities of DAGE and TAGE increase with an increase of catalyst amount, whereas the selectivity of MAGE decreases. This can be considered that the DAGE and TAGE could be generated due to the further dehydration of MAGE with methanol.
As can be seen, during the dehydration of ethanol with glycerol or tert-butyl alcohol, the similar trends of glycerol conversion and products selectivities are presented. Increasing the amount of catalyst from 1 to $10 \%$, it leads to an enhancement of glycerol conversion, as well as for the selectivities of DAGE and TAGE. It could be due to the fact that the number of active sites increases with an increase of catalyst amount, which led to the promotion of reaction rate. The same results have been observed by previous researches..$^{38,39}$ In their works, the results obtained indicated that the increase in catalyst amount yields a higher glycerol conversion and product selectivity. In fact, the selectivities of DAGE and TAGE reach to maximum when the catalyst amount is $10 \%$.

According to the previous studies, ${ }^{24,40-42}$ the reaction mechanism between glycerol and alcohols can be described as in Figure 3.

Under acidic atmosphere, glycerol is protonated to form $\mathrm{H}_{3} \mathrm{C}^{+}-(\mathrm{OH}) \mathrm{CH}-(\mathrm{OH}) \mathrm{CH}_{2}(\mathrm{OH})$, and then it is attacked by alcohols to form MAGE, which could be protonated and reacted with alcohols again to form DAGE and TAGE. There are three methyl groups as the electron-donating group in the tert-butyl alcohol, which leads to a fact that tert-butyl alcohol is more able to react with glycerol, and the main product is MAGE. However, it is difficult that DAGE further reacts with tert-butyl alcohol to generate TAGE due to the influence of steric hindrance.

According to the above results and analysis, when the catalyst amount (wt.\% to glycerol) is over $10 \%$, it is observed that neither glycerol conversion nor product selectivities are changed significantly. It could be interpreted that the effect of acidic concentration on the dehydration of glycerol with alcohols becomes smaller when the catalyst amount increases to a certain value. Moreover, the reaction system is close to the equilibrium, and the glycerol conversion is basically unchanged.

\section{Effect of reaction temperature and time}

The glycerol dehydration with alcohols (methanol, ethanol and tert-butyl alcohol) over 10\% (wt.\% to glycerol) of A-35 was carried out under these conditions: reaction temperature of $50-10^{\circ} \mathrm{C}$, reaction time of 5-12 $\mathrm{h}, 6: 1$ molar ratio of alcohols to glycerol, and stirring speed of $70 \mathrm{rpm}$.

Effects of temperature and time on glycerol conversion in the three synthesis processes were shown in Figure 4. Effects of temperature and time on product selectivity in the glycerol dehydration with methanol were exhibited in Figure 5. Effects of temperature and time on product selectivity in the glycerol dehydration with ethanol were shown in Figure 6. Effects of temperature and time on 

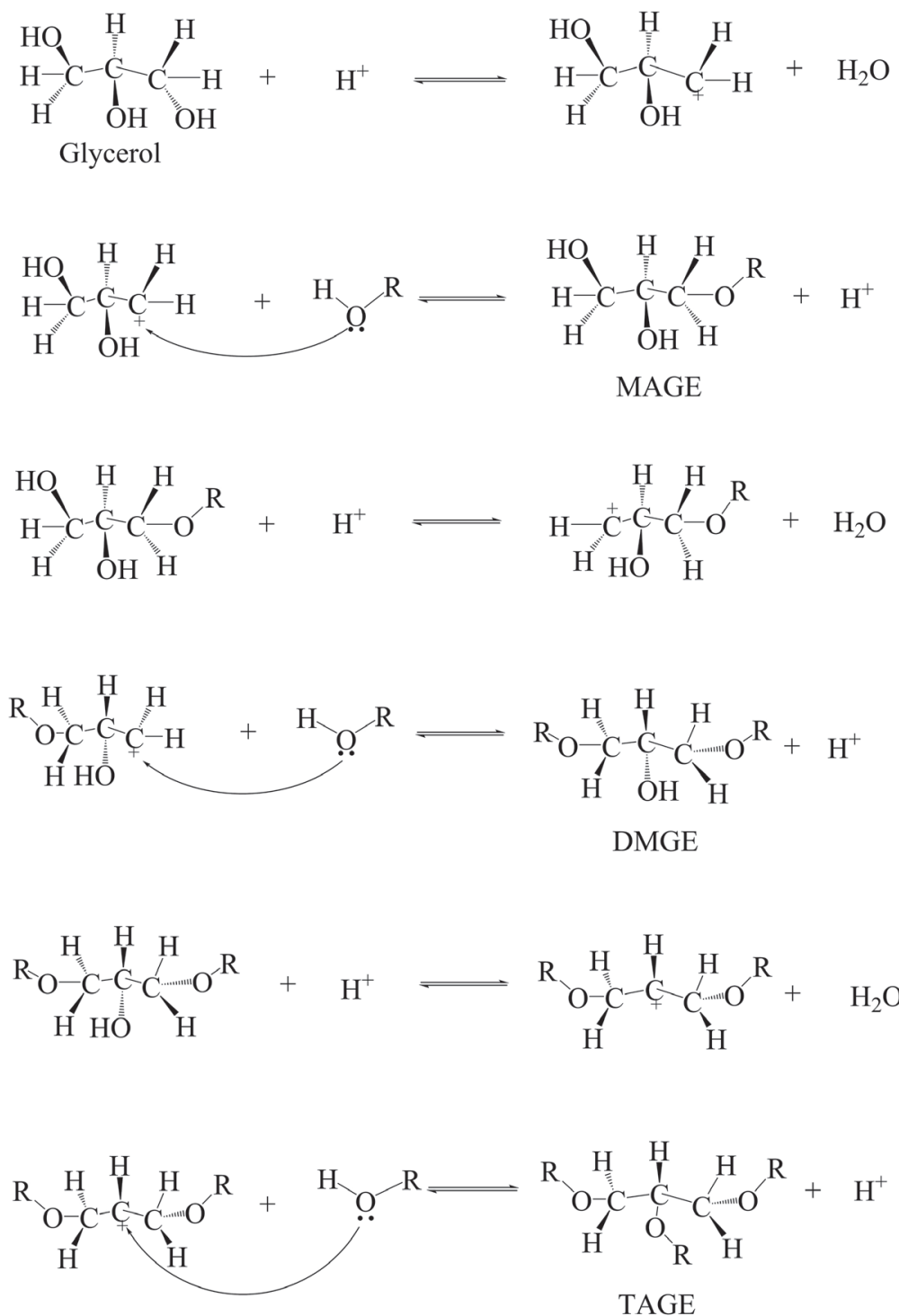

Figure 3. The reaction mechanism in dehydration of glycerol with alcohols.

product selectivity in the glycerol dehydration with tertbutyl alcohol were displayed in Figure 7.

In Figure 4, during the glycerol dehydration with alcohols at $50{ }^{\circ} \mathrm{C}$, it is seen that there is no obvious effect on the glycerol conversion with the increase of reaction time. When the temperature reaches $80{ }^{\circ} \mathrm{C}$, the reaction time presents a positive influence when it is less than $8 \mathrm{~h}$. In view of the fact that the glycerol dehydration with alcohols is an endothermic reaction, a higher reaction rate could be obtained by the increase of temperature. However, considering that the reaction is almost close to the equilibrium, the glycerol conversion maintains stable after $8 \mathrm{~h}$. During the glycerol dehydration with methanol, methanol could react with itself to generate gas-phase dimethyl ether in higher temperature, which causes a decline of the glycerol conversion.
Considering that this reaction is reversible, prolonging the reaction time can make the reaction equilibrium shift to the right side. When the reaction time increases from 8 to $12 \mathrm{~h}$, the MAGE selectivity increases gradually, and the DAGE selectivity shows a decline. This could be explained by the water from the reaction system that causes the DAGE hydrolysis with the reaction process. On the other hand, the water could be adsorbed on the catalyst acid site, ${ }^{25}$ which leads to the reduction of catalytic efficiency. When the reaction temperature is 80 or $100{ }^{\circ} \mathrm{C}$, the same trend is obtained.

As seen in Figure 6, during the glycerol dehydration with ethanol, the glycerol conversion maintains stable after $8 \mathrm{~h}$, and it does not change with the increase of temperature from 80 to $100^{\circ} \mathrm{C}$. When the reaction temperature is $80^{\circ} \mathrm{C}$, the highest selectivities of DAGE and TAGE are obtained 


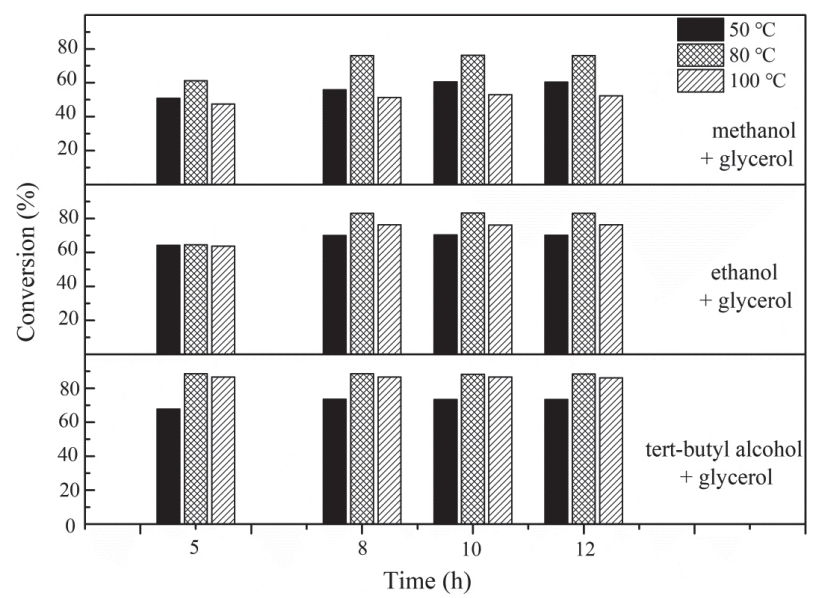

Figure 4. Effects of time course on glycerol conversion at 50-100 ${ }^{\circ} \mathrm{C}$.

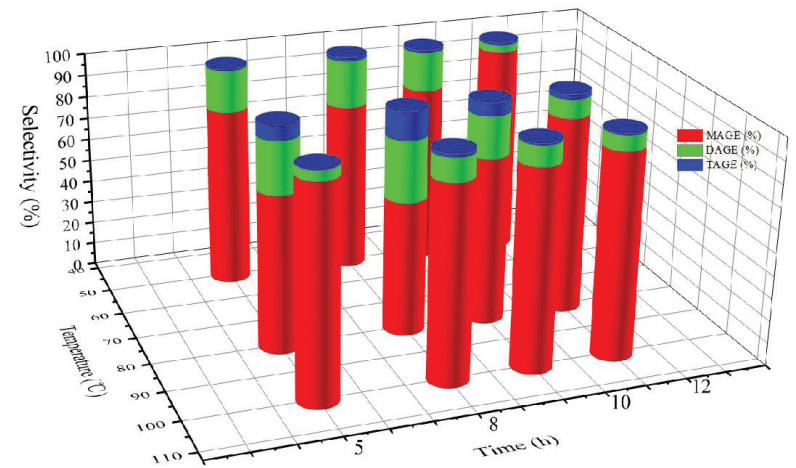

Figure 5. Effects of time and temperature on product selectivity in glycerol dehydration with methanol.

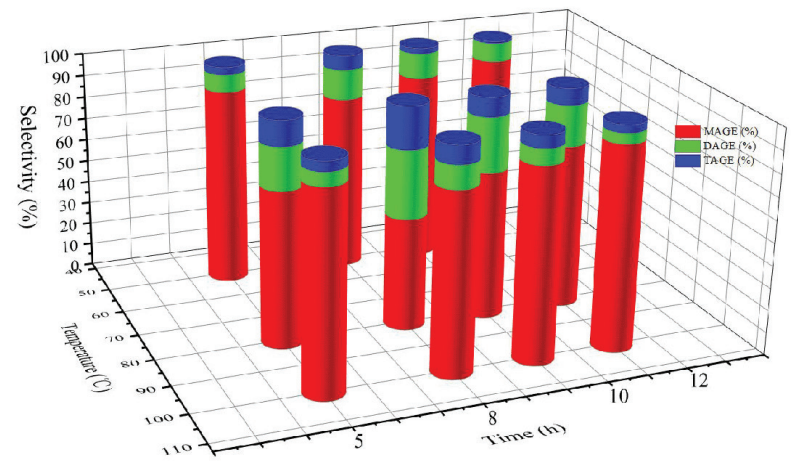

Figure 6. Effects of time and temperature on product selectivity in glycerol dehydration with ethanol.

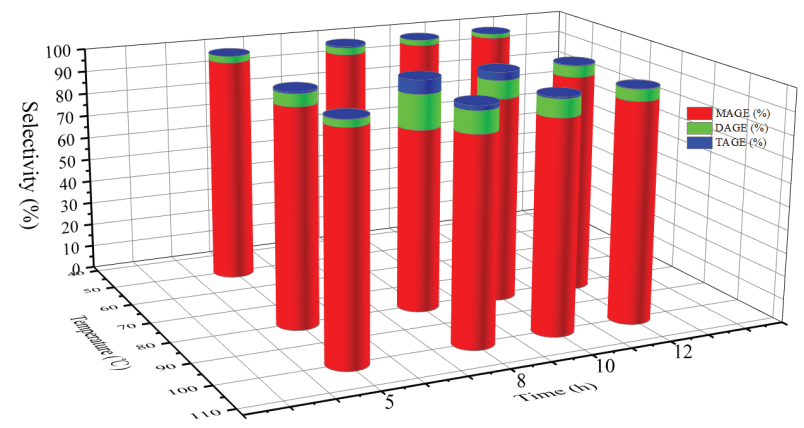

Figure 7. Effects of time and temperature on product selectivity in glycerol dehydration with tert-butyl alcohol. at $8 \mathrm{~h}$. In view of the fact that the glycerol dehydration with alcohols is an endothermic reaction, the increase of temperature can produce a higher reaction rate.

The glycerol dehydration with tert-butyl alcohol was also studied. As shown in Figure 7, the selectivity of TAGE is very low at $50{ }^{\circ} \mathrm{C}$, and it only reaches $0.7 \%$ when the reaction lasts $8 \mathrm{~h}$. This result could be caused by the influence of steric hindrance. Clearly, when the temperature is $80^{\circ} \mathrm{C}$, an increase of the glycerol conversion (Figure 4) is observed, as well as those of the DAGE and TAGE selectivities. In fact, at $80{ }^{\circ} \mathrm{C}$, the selectivities of DAGE and TAGE run up to the highest when the reaction lasts $8 \mathrm{~h}$.

Based on above results, an appropriate reaction temperature $\left(80^{\circ} \mathrm{C}\right)$ and time $(8 \mathrm{~h})$ could be in favor for the glycerol dehydration with alcohols.

\section{Effect of the feed molar ratio of alcohols to glycerol}

The glycerol dehydration with alcohols (methanol, ethanol and tert-butyl alcohol) over $2.0 \mathrm{~g}$ of ion exchange resin Amberlyst-35 was studied at reaction temperature of $80^{\circ} \mathrm{C}$, reaction time of $8 \mathrm{~h}$, and stirring speed of $70 \mathrm{rpm}$. The effect of the alcohols to glycerol molar ratio on glycerol conversion and product selectivities was shown in Figure 8.

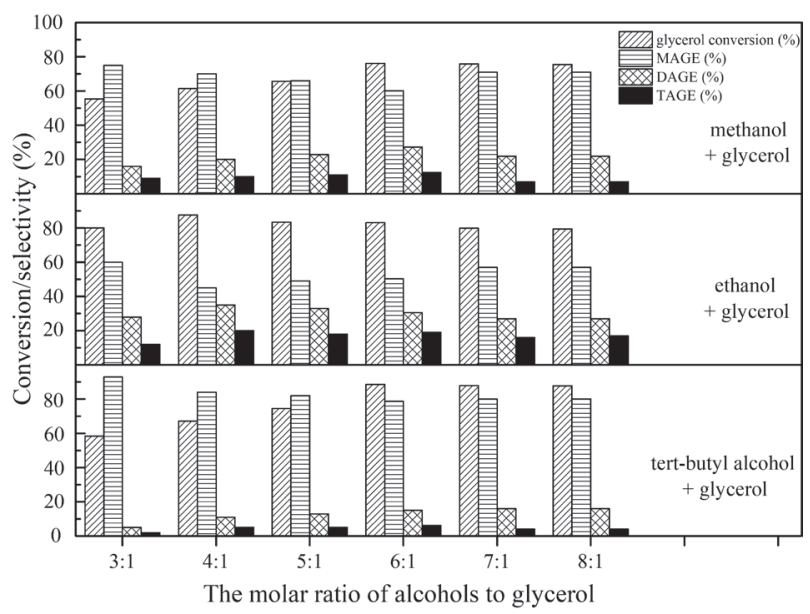

Figure 8. The effect of the alcohols to glycerol molar ratio on catalysis performance.

For the glycerol dehydration with methanol, it can be seen from Figure 8 that with an increase of the molar ratio from 3:1 to $6: 1$, the glycerol conversion and the selectivities of DAGE and TAGE increase gradually, while the selectivity of MAGE decreases. When the molar ratio of methanol to glycerol is $6: 1$, the highest conversion of glycerol, as well as the selectivities of higher ethers (DAGE, TAGE), is observed. This could be due to that an increase of the methanol amount could shift the reaction equilibrium 
toward the right side. However, the catalysis effect shows a negligible difference when the molar ratio is increased from $6: 1$ to $7: 1$ or $8: 1$.

During the glycerol dehydration with ethanol, the best result is exhibited at the 4:1 molar ratio of ethanol to glycerol. Compared with methanol, the selectivities of the higher ethers are better during the dehydration of glycerol with ethanol. The product of dehydration of glycerol with tert-butyl alcohol is mainly MAGE. The higher ethers selectivities run up to the highest when the molar ratio of tert-butyl alcohol to glycerol is 6:1, as well as the glycerol conversion.

The product distribution of MAGE, DAGE and TAGE, the effect of the alcohols to glycerol molar ratio on product yields was shown in Figure 9.

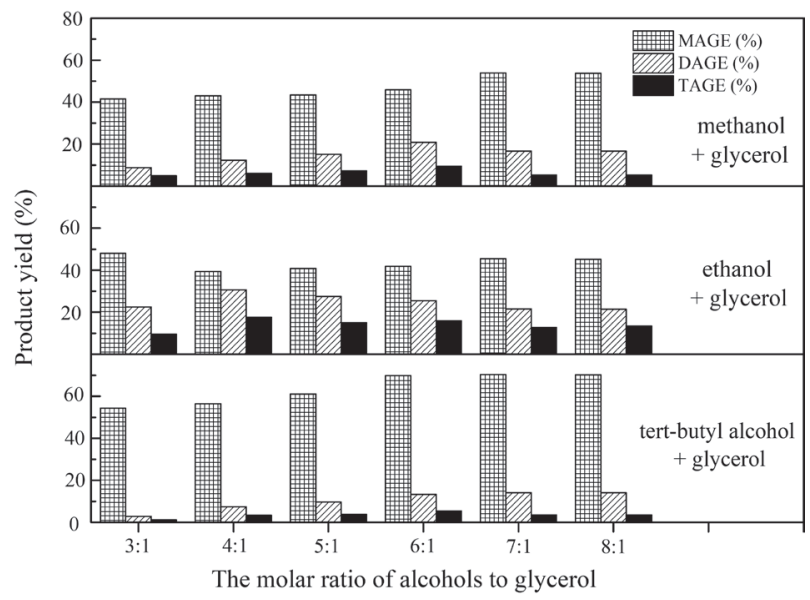

Figure 9. The effect of the alcohols to glycerol molar ratio on product yields.

Firstly, the comparative analysis for the MAGE yield of the three synthesis processes was carried out. For the glycerol dehydration with methanol, it is observed from Figure 9 that the yield of MAGE increased gradually with the increase of molar ratio from $3: 1$ to $7: 1$, and when the molar ratio is $7: 1$, the yield of MAGE is close to $60 \%$. However, for the glycerol dehydration with ethanol, the MAGE yield presents a decline when the molar ratio of raw materials increases from $3: 1$ to $4: 1$. An increase of molar ratio from 4:1 to $7: 1$ provides an increase of the MAGE yield. The MAGE yield reaches the maximum (about 50\%) at the $7: 1$ of molar ratio.

For the dehydration process of glycerol with tert-butyl alcohol, the MAGE yield is increased with the increase of molar ratio. When the molar ratio is $7: 1$, the highest MAGE yield (about 70\%) is presented because the reaction achieved equilibrium.

From the above results, it can be concluded that the glycerol dehydration with tert-butyl alcohol could be a good choice if MAGE is considered as the target product.

Secondly, the comparative analysis for the higher ether (DAGE, TAGE) yields was performed. Comparing the other two processes, the glycerol dehydration with ethanol presents the desired yields of higher ethers. With regard to glycerol and ethanol, both of them are derived from biomass and are renewable. If this route, which glycerol and ethanol are converted into the higher ethers (di- and tri-ethyl glycerol ether), is commercialized, it will promote effectively the scale application of biomass energy and the cleaner utilization of fossil fuel.

As far as the reaction system is concerned, a large amount of water is generated in the glycerol dehydration with alcohols. ${ }^{24,25,42}$ The higher the catalyst activity is, more water is produced. If the water cannot be removed from the system promptly, it will affect the progress of the forward reaction and lead to the catalyst deactivation.

In this paper, the preliminary comparative analysis of glycerol dehydration with alcohols was carried out only in the liquid phase or near liquid phase, and the technological processes adapted to commercialization need to be explored and validated in the next successive heterogeneous reaction process.

\section{Conclusions}

The dehydration of glycerol with three alcohols (methanol, ethanol and tert-butyl alcohol) over ion exchange resin Amberlyst-35 was studied. During the glycerol dehydration with alcohols, the optimum molar ratio of glycerol to methanol, ethanol and tert-butyl alcohol were $1: 6,1: 4$, and 1:6, respectively. The highest selectivities of DAGE and TAGE were observed at the optimum condition (temperature: $80^{\circ} \mathrm{C}$, time: $8 \mathrm{~h}$ ). It is evident from the data that there is a significant increase in reaction rate when the catalyst amount (wt.\% to glycerol) increased to $10 \%$, which caused a higher glycerol conversion and product selectivity. Furthermore, MAGE is obtained as the main product in glycerol dehydration with tert-butyl alcohol due to the influence of steric hindrance.

In conclusion, the glycerol dehydration with tert-butyl alcohol could be a good choice if MAGE was considered as the target product, while the glycerol dehydration with ethanol could be a better route converting biomass material into vehicle fuel in view of its desired yields of higher ethers.

\section{Acknowledgments}

The authors are grateful for the support of chemical reagents and instruments from Shanxi HuaDun Co., Ltd. 


\section{References}

1. Gaudin, P.; Jacquot, R.; Marion, P.; Pouilloux, Y.; Jérôme, F.; ChemSusChem 2011, 4, 719.

2. Davies, T. E.; Kondrat, S. A.; Nowicka, E.; Graham, J. J.; Apperley, D. C.; Taylor, S. H.; Graham, A. E.; ACS Sustainable Chem. Eng. 2016, 4, 835.

3. Wang, J.; Zhao, X.; Nian, L.; Lei, N.; Li, L.; Zhang, L.; Xu, S.; Miao, S.; Pan, X.; Wang, A.; Zhang, T.; ChemSusChem 2016, 9, 784 .

4. Naniwa, K.; Tsushima, Y.; Furuya, M.; Shirai, H.; JP pat. 20072910492007.

5. Ved, H. S.; Gustow, E.; Mahadevan, V.; Pieringer, R. A.; J. Biol. Chem. 1984, 259, 8115.

6. Haynes, M. P.; Buckley, H. R.; Higgins, M. L.; Pieringer, R. A.; Antimicrob Agents Chemother. 1994, 38, 1523.

7. Hazra, A. G.; Chatterjee, P.; Ind. Crops Prod. 2008, 27, 39.

8. Pedrono, F.; Martin, B.; Leduc, C.; Le Lan, J.; Saiag, B.; Legrand, P.; Moulinoux, J.-P.; Legrand, A. B.; Nutr. Cancer 2004, 48, 64 .

9. Deniau, A.-L.; Mosset, P.; Pédrono, F.; Mitre, R.; Le Bot, D.; Legrand, A. B.; Mar. Drugs 2010, 8, 2175.

10. Hellú, J. A. A.; Marques Neto, I.; Duque, J. C. M.; Ars Vet. 2012, 28, 209.

11. Diehl, K. H.; Eggensperger, H.; Oltmanns, P.; Scheler, K. H.; DE pat. 41404741993.

12. Neuss, M.; Albers, T.; Bruening, S.; Ansmann, A.; Gondek, H.; Schmid, K.-H.; US pat. 201002617982010.

13. Beilfuss, W.; Diehl, K. H.; Eggensperger, H.; Oltmanns, P.; DE pat. 42406741994.

14. Ishikawa, A.; Kuwano, Y.; Fujii, M.; JP pat. 20072827972007.

15. Hamashima, H.; Aono, M.; Mitamura, J.; Toda, M.; JP pat. 20011721342001.

16. Cauwet, D.; Dubief, C.; EP pat. 05551551993.

17. Kasuga, K.; Miyajima, T.; WO pat. 01321342001.

18. Beilfuss, W.; Wutsch, S.; Weber, K.; Gradtke, R.; US pat. 200802550152008.

19. Amar, D.; Cotton, T.; Lavaud, B.; FR pat. 29231592009.

20. Melero, J. A.; Vicente, G.; Morales, G.; Paniagua, M.; Moreno, J. M.; Roldán, R.; Ezquerro, A.; Pérez, C.; Appl. Catal., A 2008, $346,44$.

21. Di Serio, M.; Casale, L.; Tesser, R.; Santacesaria, E.; Energy Fuels 2010, 24, 4668.

22. Lee, H. J.; Seung, D.; Jung, K. S.; Kim, H.; Filimonov, I. N.; Appl. Catal., A 2010, 390, 235.
23. Klepáčová, K.; Mravec, D.; Kaszonyi, A.; Bajus, M.; Appl. Catal., A 2007, 328, 1.

24. Karinen, R. S.; Krause, A. O. I.; Appl. Catal., A 2006, 306, 128.

25. Pariente, S.; Tanchoux, N.; Fajula, F.; Green Chem. 2009, 11, 1256.

26. Yuan, Z.; Xia, S.; Chen, P.; Hou, Z.; Zheng, X.; Energy Fuels 2011, 25, 3186.

27. Sepúlveda, J. H.; Vera, C. R.; Yori, J. C.; Badano, J. M.; Santarosa, D.; Mandelli, D.; Quim. Nova 2011, 34, 601.

28. Lee, H. J.; Seung, D.; Filimonov, I. N.; Kim, H.; Korean J. Chem. Eng. 2011, 28, 756.

29. Ozbay, N.; Oktar, N.; Dogu, G.; Dogu, T.; Ind. Eng. Chem. Res. 2012, 51, 8788 .

30. Zhao, W.; Yang, B.; Yi, C.; Lei, Z.; Xu, J.; J. Ind. Eng. Chem. 2010, 49, 12399

31. Zhou, C.; Beltramin, J. N.; Fan, Y.-X.; Lu, G. Q.; Chem. Soc. Rev. 2008, 37, 527.

32. Frusteri, F.; Arena, F.; Bonura, G.; Cannilla, C.; Spadaro, L.; Di Blasi, O.; Appl. Catal., A 2009, 367, 77.

33. Kiatkittipong, W.; Suwanmanee, S.; Laosiripojana, N.; Praserthdam, P.; Assabumrungrat, S.; Fuel. Process. Technol. 2010, $91,456$.

34. https://cmallegro.cn.china.cn, accessed in June 2018.

35. Advanced Chemistry Development, Inc.; ACD/labs, version 2016/14.0; Advanced Chemistry Development, Inc., Toronto, Canada, 2016.

36. CambridgeSoft Corp.; ChemBioOffice, version 2014; CambridgeSoft Corp., Britain, 2014.

37. Klepáčová, K.; Mravec, D.; Bajus, M.; Appl. Catal., A 2005, 294, 141.

38. Ikizer, B.; Oktar, N.; Dogu, T.; Fuel. Process. Technol. 2015, 138,570 .

39. Gonzalez-Arellano, C.; Grau-Atienza, A.; Serrano, E.; Romero, A. A.; Garcia-Martinez, J.; Luque, R.; J. Mol. Catal. A: Chem. $\mathbf{2 0 1 5}, 406,40$.

40. da Silva, C. R. B.; Gonçalves, V. L. C.; Lachter, E. R.; Mota, C. J. A.; J. Braz. Chem. Soc. 2009, 20, 201.

41. Gordiyenko, O. I.; Linnik, T. P.; Gordiyenko, E. O.; Bioelectrochemistry 2004, 62, 115.

42. Jin, D.; Hou, Z.; Zhang, L.; Zheng, X.; Catal. Today 2008, 131, 378.

Submitted: August 7, 2018

Published online: November 7, 2018 